\title{
DIETARY AND LIFESTYLE PRACTICES OF STROKE PATIENTS IN THE YEAR FOLLOWING STROKE
}

\author{
Leeanne Carey ${ }^{1,2}$, Elizabeth V Harris ${ }^{1}$, Tamara Tse ${ }^{1,2}$ \\ and the START cohort team \\ ${ }^{1}$ Neurorehabilitation and Recovery, \\ Florey Institute of Neuroscience and Mental Health \\ ${ }^{2}$ Occupational Therapy, School of Allied Health, La Trobe University
}

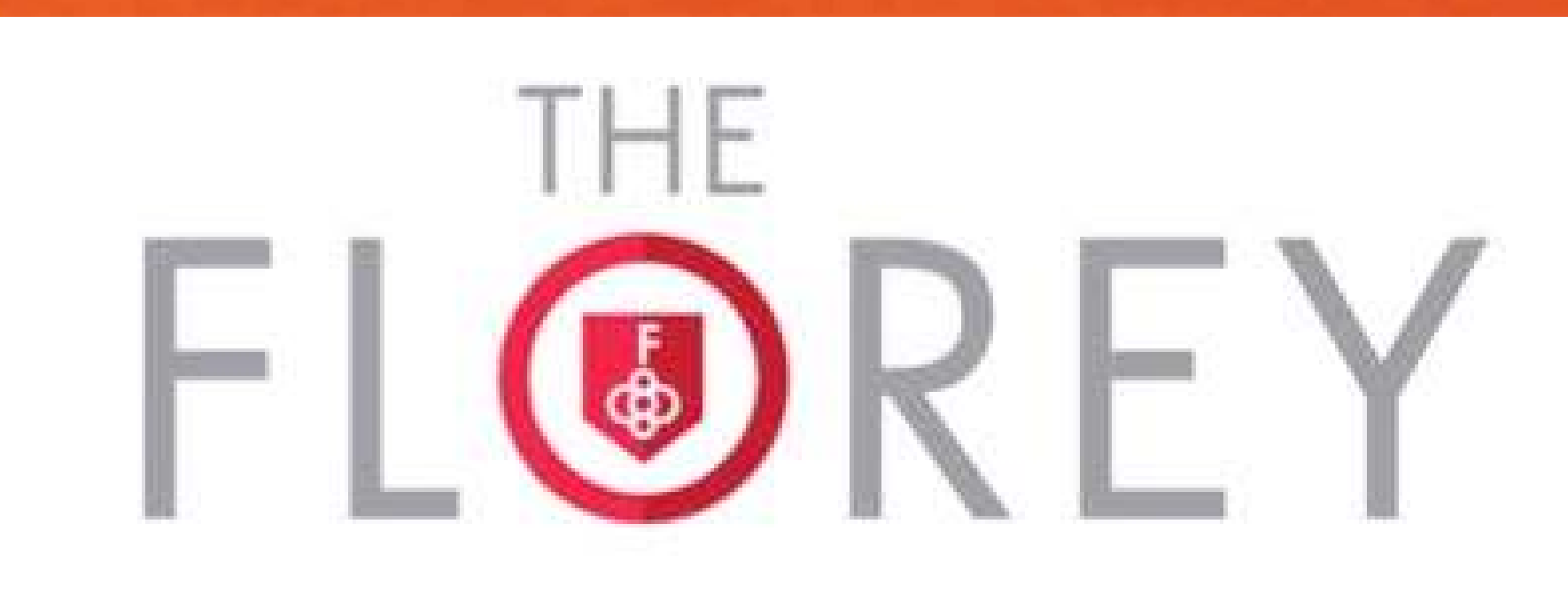

\section{BACKGROUND AND AIMS}

Dietary and lifestyle factors are major contributors to the incidence and burden of stroke. ${ }^{1,2}$ Stroke survivors have a high rate of recurrent stroke with up to $19 \%$ of patients experiencing a secondary stroke within 5 years. ${ }^{3}$ Guidelines from the Stroke Foundation ${ }^{4}$ recommend that stroke patients improve their diet and lifestyle to prevent recurrent stroke. However, few studies have reported the diet and lifestyle behaviours of stroke patients under usual care.

Aim: to characterize the dietary habits, lifestyle behaviours, and related metabolic risk factors of stroke patients longitudinally, i.e. prior to stroke/baseline (assessed at initial hospital stay) and at 3 months and 12 months post stroke.

\section{METHODS}

The START study (STroke imAging pRevention and Treatment) was a multi-site longitudinal observational cohort study of patients with ischemic stroke. 5,6

\section{Measures}

Patients completed the Cancer Council Food Frequency Questionnaire, ${ }^{7}$ the Rapid Assessment of Physical Activity (RAPA), ${ }^{8}$ and additional questions relating to diet and lifestyle behaviours. Height, weight and waist circumference were measured.

Patients met recommendations if they were:

- not currently smoking

- eating a nutritionally balanced diet

- $\geq 5$ portions fruit/vegetables daily

- not adding salt to food

- $\leq 2$ standard drinks per day $\leq 4$ standard drinks on any occasion

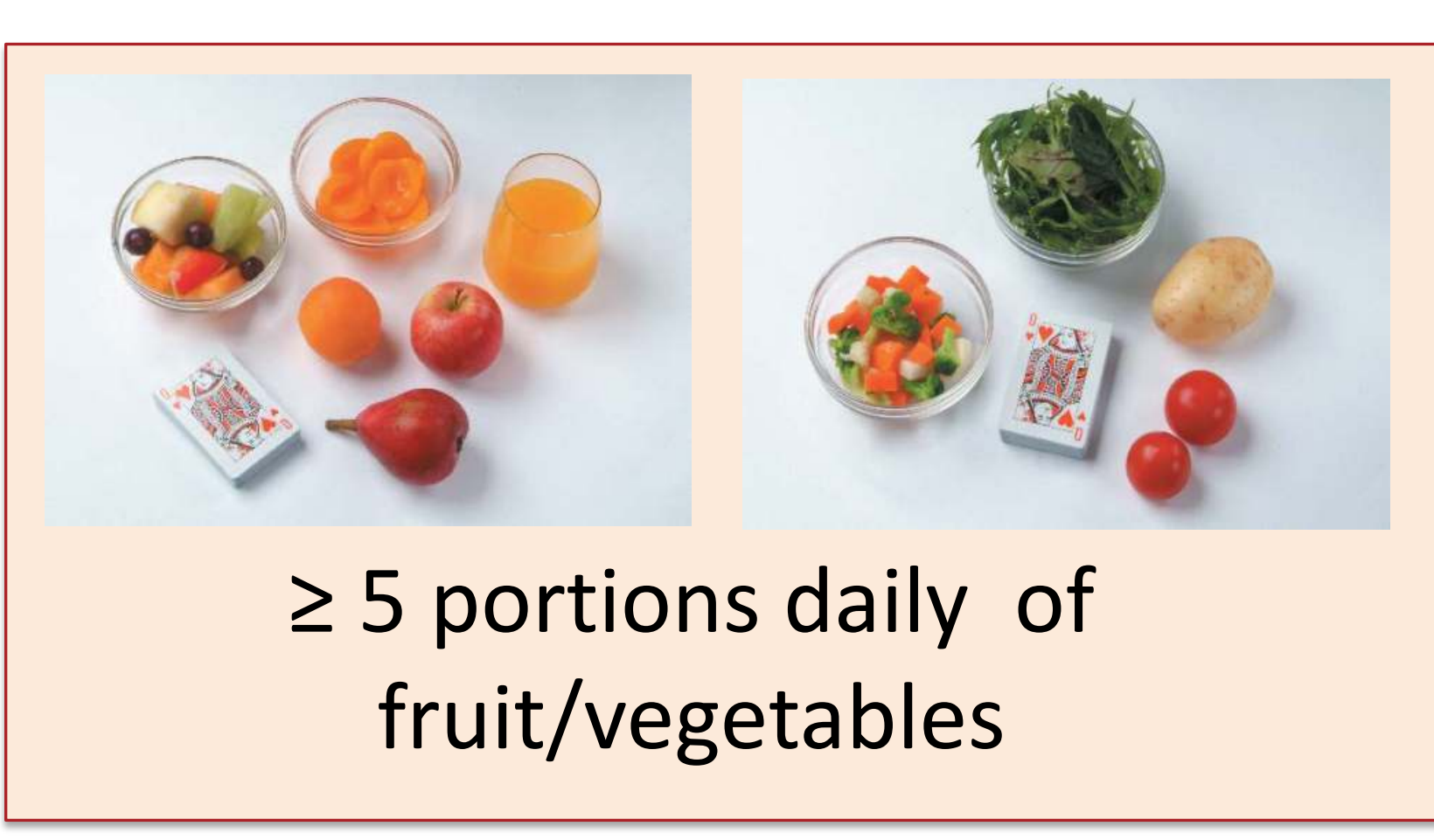

- $\geq 2.5$ hours of moderate intensity physical activity per week

- Body Mass Index (BMI) $\left(\mathrm{kg} / \mathrm{m}^{2}\right)<25$

- waist circumference $\leq 80 \mathrm{~cm}$ (females) or $\leq 94 \mathrm{~cm}$ (males)

\section{Statistical analyses}

Frequency and percentage of patients meeting Stroke Foundation recommendations are reported.

McNemar's tests were used to determine significant differences in the proportion of patients meeting recommendations between baseline and 12 months.

\section{RESULTS}

Participants included 219 stroke patients aged 27-95 years, with a mean age of 69 years. Nearly half had suffered a mild stroke (Table 1). Compared with females, males were younger, had experienced less severe stroke, and met dietary and lifestyle guidelines less frequently.

\section{Table 1. Patient characteristics at baseline.}

\begin{tabular}{|llcc|} 
Age & & $n$ & $\%$ \\
& $<65$ years & 77 & 35.2 \\
Gender & $\geq 65$ years & 142 & 64.8 \\
& Male & 140 & 63.9 \\
NIHSS Score & Female & 79 & 36.1 \\
(day 3-7) & Mild $\leq 5$ & 116 & 81.7 \\
& Moderate 6-13 & 16 & 11.3 \\
\hline
\end{tabular}

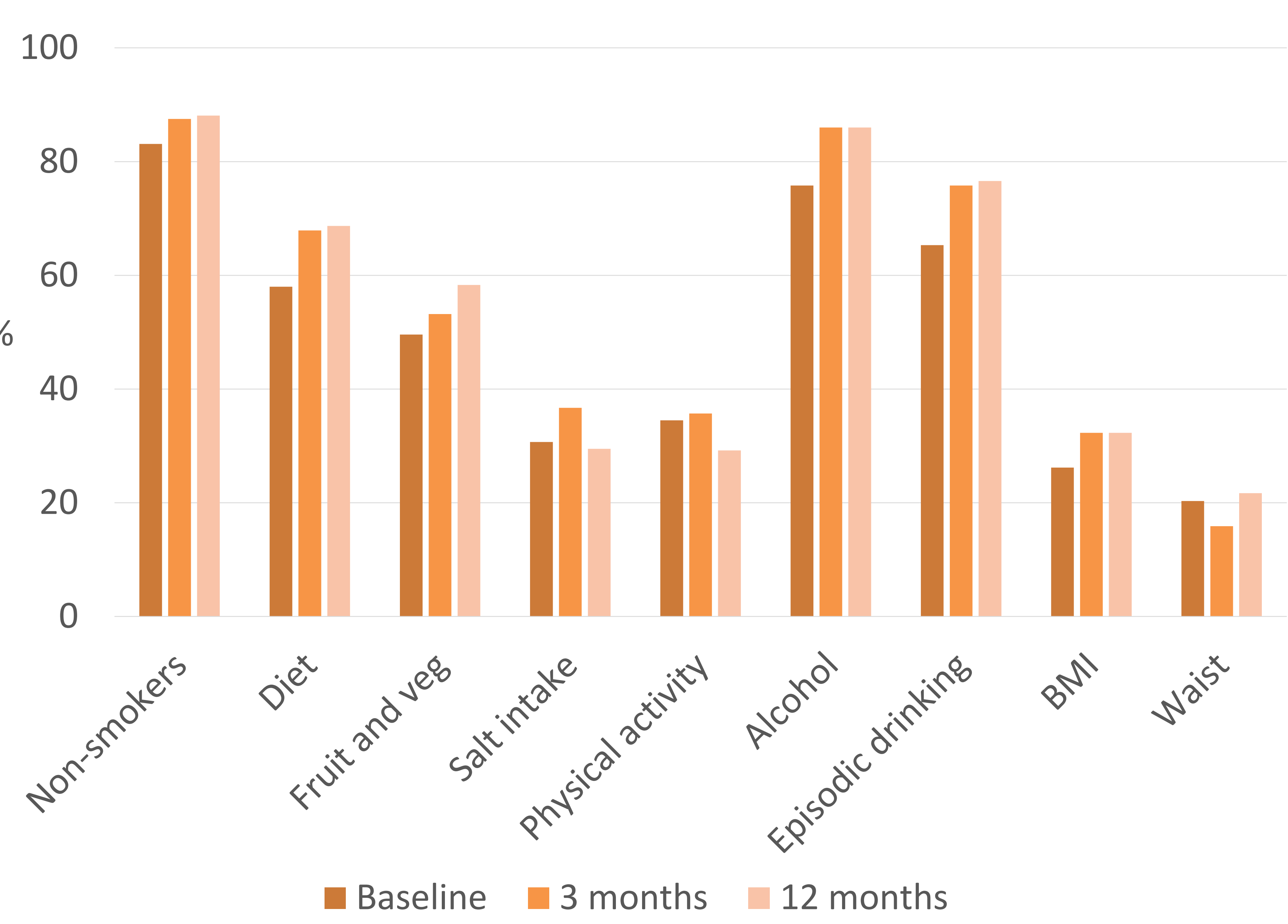

Figure 1. Percentage of stroke patients meeting recommendations at baseline, 3 months and 12 months.

At baseline, most patients were non-smokers and met recommendations for alcohol consumption. BMI and waist circumference had the fewest participants in the healthy range. Less than one half of patients met dietary guidelines and only a third obtained sufficient exercise.

The percentage of patients meeting guidelines did not change substantially from baseline to 3 - and 12 months for any of the health measures. Significant 12-month differences were only observed for daily alcohol consumption.

\section{DISCUSSION}

Stroke patients in the START study showed poor adherence to dietary and lifestyle recommendations at the time of their stroke, with only very small improvements over the following 12 months.

Patients receive information on improving their lifestyle during their hospital stay. ${ }^{9}$ Despite these recommendations from the Stroke Foundation, few patients achieve optimal behaviours, particularly for diet, physical activity and obesity. This places them at risk for further stroke and other cardiovascular events. ${ }^{3}$

\section{CONCLUSIONS}

This study highlights the need for more effective diet and lifestyle modification strategies in stroke patients.

\section{REFERENCES}

1. Feigin, V.L., et al., 2014. Global and regional burden of stroke during 1990-2010: Findings from the Global Burden of Disease Study 2010. The Lancet, 383(9913), 245-255.

2. O'Donnell, M.J., et al., 2016. Global and regional effects of potentially modifiable risk factors associated with acute stroke in 32 countries (INTERSTROKE): a case-control study. The Lancet, 388(10046), 761-775.

3. Benjamin, E.J., et al., 2017. Heart Disease and Stroke Statistics 2017 Update: A Report from the American Heart Association. Circulation, 135(10), e146-e603.

4. Stroke Foundation, Clinical Guidelines for Stroke Management, 2017: Melbourne Australia.

5. Carey, L.M. et al., 2015. STroke imAging pRevention and treatment (START): A longitudinal stroke cohort study: Clinical trials protocol International Journal of Stroke, 10(4), $636-644$.

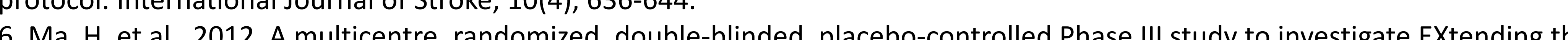
time for Thrombolysis in Emergency Neurological Deficits (EXTEND). International Journal of Stroke, 7(1), 74-80.

7. Giles G.G., Ireland P.D., 1996. Dietary Questionnaire for Epidemiological Studies (V2). Melbourne: Cancer Council Victoria.

8. Topolski, T.D. et al. 2006. The Rapid Assessment of Physical Activity (RAPA) among older adults. Preventing Chronic Disease, 3(4), A118.

9. Stroke Foundation, My Stroke Journey. A resource for stroke survivors and their carers, 2017. 